\title{
Reflets
}

Revue ontaroise d'intervention sociale et communautaire

\section{Les mémoires et les stages de maîtrise en service social}

Claude Yvette Akoun, Mélanie Aylen, Renée J. Barbeau, Nadine Blais, Véronique Bouchard, Nathalie Bouffard, Carli-Anne Borysenko, Christine Côté, Isabelle Delisle, Marilyn Desrosiers, Marlène Doepner, Renée-Maude Gravel, Armand Kayolo, Christiane Kheirallah, Janelle Lalonde, Nadia Lauzon, Marie-Josée Legault, Isabelle Légère, Renée C. Mallet, Léna Diamé Ndiaye, Florence Ngenzebuhoro, Sylvie Paiement, Hélène Petit et Myriam Rousseau

Volume 9, numéro 2, automne 2003

Travail et mieux-être

URI : https://id.erudit.org/iderudit/011101ar

DOI : https://doi.org/10.7202/011101ar

Aller au sommaire du numéro

Éditeur(s)

Reflets : Revue ontaroise d'intervention sociale et communautaire

ISSN

1203-4576 (imprimé)

1712-8498 (numérique)

Découvrir la revue

Citer ce document

Akoun, C. Y., Aylen, M., Barbeau, R. J., Blais, N., Bouchard, V., Bouffard, N., Borysenko, C.-A., Côté, C., Delisle, I., Desrosiers, M., Doepner, M., Gravel, R.-M., Kayolo, A., Kheirallah, C., Lalonde, J., Lauzon, N., Legault, M.-J., Légère, I., Mallet, R. C., Ndiaye, L. D., Ngenzebuhoro, F., Paiement, S., Petit, H. \& Rousseau, M. (2003). Les mémoires et les stages de maîtrise en service social. Reflets, 9(2), 254-280. https://doi.org/10.7202/011101ar

Tous droits réservés @ Reflets : Revue ontaroise d'intervention sociale et communautaire, 2002
Ce document est protégé par la loi sur le droit d'auteur. L'utilisation des services d'Érudit (y compris la reproduction) est assujettie à sa politique d'utilisation que vous pouvez consulter en ligne.

https://apropos.erudit.org/fr/usagers/politique-dutilisation/ 


\section{Éducation familiale sous tension : les parents immigrants francophones originaires de l'Afrique subsaharienne au Québec et l'éducation de leurs adolescents}

\section{Claude Yvette Akoun, École de service social, Université d'Ottawa}

Le présent ouvrage vise à examiner comment les auteurs ont analysé la problématique des conflits intergénérationnels entre parents immigrants et leurs adolescents. Pour ce faire, nous avons choisi de nous appuyer sur le cas des parents immigrants francophones originaires d'Afrique subsaharienne et habitant au Québec à cause, d'une part, de mon statut de mère et citoyenne québécoise originaire de Côte d'Ivoire et, d'autre part, du peu d'études sur les familles immigrantes qui portent sur les familles en provenance d'Afrique subsaharienne.

Pour répondre à notre objectif de recherche, nous avons d'abord décrit le contexte dans lequel se déroulent les conflits parents-adolescents, faisant le constat de ces conflits et établissant le profil de l'immigration africaine au Canada et au Québec. Nous avons ensuite effectué une recension des analyses portant sur les conflits intergénérationnels avant d'examiner les conséquences sur l'intervention des différents facteurs explicatifs développés dans ces analyses.

Notre analyse a montré que les facteurs à l'origine des conflits intergénérationnels entre parents immigrants et leurs adolescents sont divers et complexes. Il en ressort aussi que les facteurs explicatifs liés à la différence culturelle dominent dans les analyses qui émanent des milieux de recherche et d'intervention sociale au Québec. L'analyse a donc signalé l'importance d'une intervention axée aussi sur d'autres dimensions d'analyse affectant 
les conflits entre parents et adolescents, en particulier les facteurs structurels. Nous suggérons alors quelques modèles d'intervention appropriés.

\section{«Malade mental et criminel». Analyse de la trajectoire des individus qui vivent avec la double étiquette}

Mélanie Aylen

École de service social, Université d'Ottawa

Le but de notre mémoire est d'explorer les liens qui existent entre les systèmes psychiatrique et judiciaire par l'entremise de l'étude d'individus «doublement étiquetés» par leur double trajectoire à travers ces deux importantes institutions de contrôle social. Il s'agit d'analyser le parcours de ces personnes à la lumière de deux grandes positions: la médicalisation de la déviance, soit le transfert de gens du système de justice vers le système de santé mentale et la substitution du traitement à la punition, et la criminalisation de la maladie mentale, soit la question de l'incarcération des psychiatrisés à l'intérieur du système de justice. La théorie de l'étiquetage, qui se penche sur les mécanismes qui nous permettent de nommer, définir et interpréter une situation, nous sert de lunette théorique alors que nous cherchons à connaitre les liens et les échanges qui existent entre les deux systèmes, à identifier certains facteurs qui favorisent un parcours plutôt qu'un autre, à analyser les conséquences de telles trajectoires pour les gens concernés, et finalement à déterminer si l'on «criminalise» davantage les gens «malades» ou si l'on «médicalise» plutôt les comportements déviants ou «criminels». Notre analyse propose que la gestion actuelle des gens doublement étiquetés semble être caractérisée par la réduction des écarts entre les systèmes psychiatrique et judiciaire. Malgré leurs objectifs officiels, il semble que dans la pratique réelle, ces deux systèmes cherchent 
à assurer le contrôle social d'individus considérés «dérangeants» tout en protégeant la société contre les risques que ces derniers peuvent représenter. En parvenant à une meilleure compréhension du phénomène, nous sommes en mesure de proposer certaines pistes pour le renouvellement des pratiques sociales à l'égard des personnes qui vivent avec la double étiquette.

\section{Quel sens donnent les femmes à la prise en charge d'un adulte dépendant et/ou en perte d'autonomie?}

\section{Renée J. Barbeau \\ École de service social, Université d'Ottawa}

L'objet de ce mémoire est d'explorer l'expérience de femmes francophones ayant comme responsabilité la prise en charge de leur mère âgée. Il s'agit donc de vérifier s'il existe vraiment un côté positif à cette expérience, c'est-à-dire, de savoir ce que les femmes retirent du rôle de personne soutien auprès de leur mère âgée dépendante et en perte d'autonomie. La personne soutien est définie ici comme la personne principale responsable d'offrir l'aide et les soins nécessaires à la personne aidée dans ses activités de vie quotidienne.

L'approche méthodologique de type qualitatif a été privilégiée pour les fins de cette étude afin de nous permettre de mieux comprendre la réalité des femmes ayant la responsabilité de la prise en charge de leur mère âgée. Pour présenter cette réalité, quatre femmes francophones ont été interviewées à l'aide d'une grille pour des entrevues semi-dirigées comportant des questions ouvertes. Ce guide a permis à ces femmes de nous faire part de leur réalité en tant que fille et soutien pour leur mère âgée ainsi que de connaître leur vécu quotidien. Pour ce qui est des points positifs soulevés, certaines femmes ont fait ressortir le sentiment d'être aimées et appréciées de leur mère et d'être soutenues par la famille. D'autres ont dit que cette tâche leur a accordé la possibilité 
de développer une meilleure solidarité entre leurs frères et soeurs et de vivre des moments privilégiés avec leur mère. Les motifs sont catégorisés en six grands concepts dont: l'aide reçue et/ou donnée, l'obligation filiale, le rôle assigné et/ou assumé, le rang dans la famille, la socialisation, l'affection et l'échange. Ces femmes expliquent que l'aide reçue de la famille et/ou du réseau formel a rendu cette expérience plus positive et que sans ce soutien, aucune n'aurait poursuivi cette tâche de personne soutien.

\section{Aborder la question des agressions sexuelles auprès des femmes immigrantes : des pratiques en mouvement}

\section{Nadine Blais}

École de service social, Université d'Ottawa

Depuis quelques années le Centre d'aide et de lutte contre les agressions sexuelles (CALAS), qui lutte contre les agressions sexuelles et vient en aide aux femmes survivantes d'agressions sexuelles en Outaouais, se préoccupe du sort des femmes immigrantes car il n'arrive pas à les rejoindre. De plus, l'Outaouais représente la troisième région d'accueil des nouveaux arrivants et des nouvelles arrivantes au Québec. Sachant que les femmes immigrantes et des communautés ethnoculturelles représentent un groupe vulnérable aux agressions sexuelles parce qu'elles sont plus isolées, victimes de racisme et de discrimination et peu outillées face à leurs droits et à leurs recours, le Regroupement québécois des CALACS ainsi que le CALAS veulent pour mieux cerner les besoins de ces femmes et y répondre plus adéquatement. Ils ont choisi d'orienter des recherches et des actions dans ce sens.

Afin de comprendre différentes pratiques d'intervention auprès des femmes immigrantes et de les intégrer à sa propre pratique d'intervention le CALAS a procédé à une recherche qualitative. Cette recherche effectuée à l'aide d'entrevues semi-structurées 
auprès de douze intervenantes expérimentées auprès des immigrantes démontre que leurs interventions, dans un contexte de violence sexuelle et conjugale, different sur plusieurs plans de l'intervention féministe «classique». Bien que la finalité de ces pratiques innovatrices et en émergence convergent vers celle de l'intervention féministe telle que pratiquée dans les groupes féministes du Québec, les interventions décrites ici tiennent compte d'aspects propres au vécu des femmes immigrantes: parcours d'immigration, méconnaissance de leur statut et leurs droits, présence importante de leur communauté et croyances religieuses. Les participantes de notre étude nous démontrent la possibilité d'un jumelage entre les objectifs et les outils féministes et celles des approches interculturelles.

Finalement notre cadre théorique ainsi que les entrevues nous auront appris l'importance fondamentale d'inclure les femmes immigrantes au sein des organismes pour penser différemment l'intervention, pour enrichir l'équipe de travail majoritairement constituée de femmes nées au Québec et surtout pour lutter contre le racisme encore présent dans la société et dans les milieux d'intervention.

\section{Le suicide dans la Ville du Grand Sudbury}

\section{Véronique Bouchard}

École de service social, Université Laurentienne

Ce mémoire examine la problématique du suicide dans la Ville du Grand Sudbury. Pour ce faire, nous présentons d'abord la théorie sociologique du suicide développée par Émile Durkheim. Ses recherches ont montré que le suicide n'est pas un acte isolé de découragement posé par un individu, mais un phénomène social qui répond à des régularités et qui se produit de manière prévisible. La perspective sociologique montre l'importance de la cohésion sociale et de l'intégration comme moyens de prévention du suicide. 
Grâce aux données épidémiologiques fournies par le Service de police de laVille du Grand Sudbury sur les décès par suicide, il a été possible de présenter un portrait du suicide dans cette communauté pour la période allant de 1992 à 2002. L'échantillon de 256 suicides permet de montrer certaines caractéristiques personnelles, sociales et circonstancielles dans la vie des personnes qui se sont suicidées. On a aussi pu établir la fréquence selon l'année, la région, l'âge, le sexe, les méthodes utilisées, les facteurs d'influence sur le comportement ainsi que les notes de suicide.

Les résultats de l'analyse du suicide dans la Ville du Grand Sudbury susciteront, nous l'espérons, une concertation et une canalisation des énergies vers une stratégie de prévention du suicide qui répondra à la nature du problème suicidaire dans cette communauté. Le défi sera de tenir compte des efforts pour diminuer les facteurs de risque et augmenter les facteurs de protection dans l'élaboration d'un plan d'action pour la mise en œuvre d'un programme de prévention pour la communauté de la Ville du Grand Sudbury.

\title{
Analyse critique d'un projet pilote expérimental implanté au sein d'une Association de famille et d'amis touchés par la maladie mentale dans le Nord- est ontarien
}

\begin{abstract}
Nathalie Bouffard,
École de service social, Université Laurentienne

Ce document porte sur un projet de stage pratique avancé qui comprenait trois objectifs: assurer l'implantation d'un programme d'information et, plus précisément, le «Programme Information Famille» (PIF), au sein de l'Association de familles et d'amis touchées par la maladie mentale; superviser un projet pilote expérimental et présenter une analyse critique du programme PIF. Ce programme a visé des familles, anglophones et francophones, dont un proche
\end{abstract}


est atteint d'une maladie mentale sévère, dans le Nord-est ontarien. Il prend en compte le rôle des familles et des amis dans le système en santé mentale. Le projet pilote répond également aux attentes du Ministère de la santé qui croit en une implication plus importante des familles et des bénévoles dans le traitement des personnes atteintes d'une maladie mentale sévère.

Le programme PIF a été développé en 1997, par Luce Fortin, originaire de Val-Rita en Ontario et employée de l'Apogée de Hull, dans la province du Québec. Comme il s'agit d'une approche spécifique, il était important de bien connaitre ce modèle et de vérifier s'il existait des études méthodologiques permettant de connaitre le succès de ce programme auprès des familles. En optant pour une approche qualitative, il a été possible d'inclure les expériences de vie et de laisser s'exprimer les familles et les amis sur un sujet donné. Compte tenu du peu de recherche au sujet de ce programme spécifique et de sa nouveauté, nous avons procédé de façon exploratoire et privilégier une certaine souplesse méthodologique. Le document présente notre réflexion théorique et pratique sur le programme.

\section{Des jeunes filles qui deviennent des parents : les trajectoires des jeunes mères qui ont peu de soutien}

\section{Carli-Anne Borysenko \\ École de service social, Université d'Ottawa}

Les conditions des femmes qui ont des enfants hors du mariage se sont transformées à travers le temps. Aujourd'hui, la pauvreté et le peu de soutien offert par les différents secteurs de la société influencent la qualité de vie des jeunes mères. Cependant, peu de recherches se concentrent sur les conditions de vie des mères adolescentes qui ont peu ou pas de soutien. En effet, la majorité des études mettent l'accent sur les jeunes mères qui reçoivent beaucoup de soutien formel par le biais des programmes et du soutien informel de leur partenaire ou de leur famille. 
Nous avons examiné trois programmes qui ciblent les jeunes mères et qui se situent dans différents secteurs d'Ottawa: Ça Mijote, Monday Place Group et la Maison Ste-Marie. Nous les avons choisis parce que chacun met l'accent sur l'un des aspects suivants, importants pour cette clientèle: la santé, le logement et le rôle du partenaire. Nos données proviennent d'une analyse des documents décrivant les programmes, ainsi que des rencontres avec les intervenantes responsables de ces derniers et d'une observation du déroulement de chaque programme.

Nos résultats indiquent que les jeunes mères reçoivent $\mathrm{du}$ soutien formel des trois programmes. Chacun leur offre des formes de soutien tangible (billets d'autobus et service de garde), affectif (interaction sociale) et informatif (cours éducationnel). Un des programmes offre du logement et un autre donne du soutien aux partenaires. Les trois programmes mettent l'accent sur la santé du bébé et sur celle de la mère, plutôt que de traiter l'aspect «adolescent» de ces jeunes mères. On favorise peu la transition de ces programmes à la vie dans la communauté, car ces derniers sont à court terme.

En conclusion, même s'il existe des programmes utiles pour jeunes mères à Ottawa, ceux-ci ne semblent pas répondre à tous les besoins exprimés par ces dernières. Cette étude conclut qu'il serait important d'offrir des programmes qui soutiennent ces jeunes mères dans leur rôle de mère et dans leur rôle d'adolescente.

\section{Le secret «barbare». Une analyse documentaire sur les grossesses suite à un viol}

\section{Christine Côté}

École de service social, Université d'Ottawa

L'historique de l'évolution des lois canadiennes sur les agressions à caractère sexuel permet de retracer les valeurs et les attitudes entretenues par la société concernant les femmes et les hommes ainsi que les rapports de pouvoir qui existent entre eux. Les 
pressions, mises de l'avant par les groupes féministes afin de revoir les lois sur le viol, n'ont pas eu les résultats souhaités. C'est ainsi qu'une des principales critiques féministes soutient que l'État maintient le rapport de pouvoir entre les sexes et qu'il valorise le maintien des valeurs patriarcales.

Une des conséquences potentielles du viol est la grossesse. Par contre, il existe très peu d'écrits à ce sujet. Les conséquences d'une grossesse suite à un viol, sur le vécu de la femme, sont peu connues. De plus, les ressources disponibles pour ces femmes sont soit méconnues ou encore, sujettes à des controverses. Ce sont les viols en temps de guerre ainsi que les conséquences des grossesses suite à ces agressions qui semblent être le plus documentés dans la littérature. Plus récemment, cette problématique a réussi à faire l'objet d'une attention internationale.

Ce mémoire se veut donc un survol de la problématique des grossesses suite à un viol. Son objectif est de démontrer, à l'aide d'une analyse féministe, comment les rapports de pouvoir entre les hommes et les femmes en Occident ont un impact sur la non reconnaissance d'une problématique, en utilisant comme exemple les grossesses suite à un viol.

\title{
De la méfiance à l'ouverture. La collaboration entre l'école et la famille : un défi de taille
}

\begin{abstract}
Isabelle Delisle
École de service social, Université d'Ottawa

Depuis quelques années, on note un intérêt marqué, tant du côté politique que de la recherche, pour évaluer et développer le partenariat entre l'école et la famille, notamment comme avenue permettant de mieux répondre aux besoins grandissants des élèves. La présente étude s'intéresse à l'identification des facteurs qui influencent la collaboration entre l'école et la famille et qui y contribuent. Dans un premier temps, un rappel historique de
\end{abstract}


l'évolution des rapports entre ces deux institutions est présenté. La problématique associée aux enjeux des rapports entre parents et écoles est ensuite située dans une perspective écologique, permettant d'évaluer les interactions complexes entre les différents systèmes impliqués dans une démarche de collaboration. L'analyse documentaire utilisée pour cette recherche porte sur trente-quatre études et articles scientifiques publiés depuis 1994. Les résultats de cette analyse comportent quatre thèmes principaux: les avantages à collaborer, les obstacles liés à cette collaboration, les facteurs facilitant l'établissement de partenariat entre l'école, la famille et la communauté ainsi que le rôle des travailleurs sociaux dans l'actualisation de ce partenariat. Enfin, l'interprétation de ces résultats débouche sur des pistes d'intervention pour la pratique, l'élaboration des politiques sociales et la recherche future.

\section{Dans l'intérêt de qui? Une analyse du concept de meilleur intérêt de l'enfant}

Marilyn Desrosiers

École de service social, Université d'Ottawa

"Agir dans le meilleur intérêt de l'enfant» semble impliquer que les professionnels des services sociaux qui s'appuient sur ce concept font référence à des notions dont la portée et la signification sont clairement établies. Or, cette notion est très vague et difficile à définir. Le but de ce mémoire est d'explorer les écrits portant sur le meilleur intérêt de l'enfant afin d'identifier les concepts qui le sous-tendent et d'analyser leur signification dans le cadre de certaines pratiques liées au bien-être à l'enfance. Nous présentons donc les résultats d'une analyse documentaire portant sur la notion de meilleur intérêt de l'enfant.

Dans un premier temps, un bref historique du concept d'enfance nous amène à constater que la notion de meilleur intérêt de l'enfant est un construit social découlant de l'évolution du statut de l'enfant depuis la fin du XIX ${ }^{\mathrm{e}}$ siècle dans les pays 
occidentaux. Par le biais d'une analyse documentaire, nous avons ensuite identifié les concepts psychologiques et sociologiques reliés à la notion de meilleur intérêt de l'enfant, soit ceux de bien-être et de besoins des enfants ainsi que les perspectives légales, notamment celles de droits des enfants et de responsabilité. La codification et l'analyse de ces concepts permettent de comprendre comment la notion de meilleur intérêt de l'enfant a évolué pour devenir une norme sociale employée pour réglementer les pratiques en service social et pour orienter des décisions légales au sujet des enfants.

De cette analyse, nous avons dégagé certaines implications sociales et politiques de la construction sociale de la norme de meilleur intérêt de l'enfant et mis en lumière quelques-unes des implications pour la pratique du service social, plus particulièrement celle dans le domaine de la protection de l'enfance.

\section{Les défis et les stratégies d'intégration des familles immigrantes au Canada}

Marlène Doepner

École de service social, Université d'Ottawa

Ce mémoire est consacré à une meilleure compréhension des défis auxquels font face les familles immigrantes au Canada ainsi qu'aux multiples stratégies d'intégration qu'elles ont élaborées. Très peu de recherches canadiennes et françaises se sont penchées sur ce sujet malgré le nombre de familles immigrantes sans cesse croissant.

Afin de mieux comprendre le contexte de l'immigration au Canada, nous avons passé en revue les politiques d'immigration instaurées par le gouvernement canadien. Par ce bref historique, nous avons pu percevoir que les lois sur l'immigration ont presque toujours été votées afin de répondre aux besoins économiques du pays. Les idéologies d'insertion canadiennes ont évolué dans 
ce même sens, et le multiculturalisme, qui est aujourd'hui dominant, reconnait qu'une des forces du Canada provient de sa diversité culturelle.

Peu importe les mesures mises en place par le gouvernement, les familles immigrantes rencontrent toujours des défis à leur arrivée au Canada, défis que nous avons catégorisés comme étant soit de nature concrète ou soit reliés aux différences culturelles. La première catégorie englobe les problèmes face au climat, à la barrière linguistique, aux conditions socio-économiques et au logement, alors que les défis culturels touchent plutôt l'éducation des enfants immigrants, les rôles des membres de la famille ainsi que l'interrelation qui existe entre les défis.

En ce qui concerne les stratégies d'intégration des familles immigrantes, nous avons remarqué que les services offerts par la société d'accueil et ceux donnés par des groupes ethniques sont d'une aide précieuse aux familles, mais que celles-ci élaborent quand même des stratégies personnelles telles: reconnaitre qu'il faut faire des compromis et des concessions, garder une forte cohésion familiale ou toujours garder une bonne communication au sein de la famille.

L'identification de telles stratégies est un excellent point de départ pour aider les personnes oeuvrant auprès des familles à mieux comprendre leur clientèle et à adapter leurs méthodes d'intervention. Quelques adaptations seraient de travailler davantage avec le réseau de soutien de la famille immigrante, de rendre les membres de celle-ci conscients des stratégies d'intégration qu'ils utilisent et de faire la diffusion de ces stratégies aux autres familles immigrantes. 


\section{L'impact du THADA sur la mère : "Ça te demande tellement $d^{\prime}$ énergie que $t^{\prime}$ as plus le goût de rien faire d'autre»}

\section{Renée-Maude Gravel}

École de service social, Université d'Ottawa

On reconnait encore aujourd'hui une certaine ambivalence dans la société quant à l'acceptation des personnes avec des incapacités, particulièrement lorsqu'il s'agit d'une incapacité invisible, tel le trouble d'hyperactivité avec déficit d'attention (THADA). Très répandue chez les enfants et adolescents, cette incapacité engendre divers problèmes au niveau social et comportemental, pour lesquels on consulte en service social. Pourtant, peu d'études se sont intéressées à l'impact de cette incapacité sur la famille et la mère.

Notre mémoire s'inscrit dans le cadre d'une recherche qualitative, réalisée auprès de mères de la région d'Ottawa qui ont un emploi et qui s'occupent d'un enfant âgé entre 6 et 17 ans avec le THADA. Notre étude explore l'impact du THADA sur la qualité de rôle maternel (gratifications et défis), en mettant l'accent sur les difficultés vécues et les liens possibles entre celles-ci, la situation familiale et la complexité des incapacités chez les enfants. Par l'entremise d'entrevues semi-dirigées, quarante mères dont onze Franco-Ontariennes ont été interrogées.

Les résultats de notre analyse de contenu thématique indiquent que ces mères vivent plusieurs difficultés, dont certaines étaient communes à toutes les participantes. D'autres différaient selon les incapacités spécifiques de l'enfant ou les caractéristiques familiales. Peu importe leur situation, les mères ont souligné être fatiguées et manquer de temps pour elles-mêmes, faire des sacrifices professionnels à cause des responsabilités familiales et avoir des difficultés à trouver des services qui répondent aux besoins exprimés. Elles sont souvent déçues par le manque d'expérience ou de qualification des personnes professionnelles rencontrées, en plus d'éprouver certaines difficultés par rapport aux médicaments. 
Les enfants peuvent avoir différents sous-types du THADA qui sont, dans environ $50 \%$ des cas, accompagnés d'autres désordres. Les mères dont l'enfant a le sous-type inattentif combiné à un trouble d'apprentissage vivent des difficultés d'ordre scolaire, alors que celles dont l'enfant a aussi un trouble psychiatrique se sentent isolées et jugées. Ces dernières se sentent obligées de sensibiliser leur entourage aux réalités du THADA, compte tenu de l'incompréhension sociétale. Lorsque l'enfant a le sous-type hyperactif-impulsif avec un trouble psychiatrique, les mères vivent surtout des difficultés reliées au comportement agressif et au tempérament explosif de leur enfant.

Les mères d'adolescents avec THADA appréhendent le futur pour ceux-ci et celles dont plus d'un enfant en est atteint vivent davantage de discordes familiales. Celles qui vivent en couple reçoivent peu d'aide de leur conjoint mais jouissent d'une plus grande flexibilité financière leur permettant de travailler à temps partiel, contrairement aux mères monoparentales. Le THADA demeure une incapacité complexe et mal comprise, tant dans les milieux professionnels que communautaires. Une sensibilisation s'impose pour changer les attitudes négatives dont sont victimes les mères et leurs enfants.

\section{Goffman et les ÉCTI}

Armand Kayolo

École de service social, Université d'Ottawa

La présente étude est tout d'abord une relecture de Goffman; elle explore les grandes notions socio-philosophiques utilisées par Goffman pour mettre au grand jour le fonctionnement de ce qu'il appelle institutions totalitaires et l'usage que la société nordaméricaine fait des personnes ayant un handicap physique ou mental. De l'époque des asiles jusqu'à la désinstitutionalisation psychiatrique, le déplacement de l'axe de la problématique de santé mentale ne peut pas être compris uniquement comme un 
échec des processus de désinstitutionalisation; il participe aussi aux mutations profondes de la société.

Notre étude met, dans un deuxième temps, l'accent sur une nouvelle forme d'organisation psychiatrique, les équipes communautaires de traitement intensif (ÉCTI). Les ÉCTI proposent aux malades mentaux graves une relation d'aide pour surmonter les difficultés qui surgissent dans leur quotidien et qui nuisent à leur intégration sociale. Les ÉCTI ont donc la potentialité de tout mettre en œuvre pour sortir ces personnes de l'exclusion institutionnelle, dénoncée par Goffman. Mais en quoi, aujourd'hui, un apport de la pensée de Goffman peut-il être pertinent dans l'analyse et la compréhension des ÉCTI? Il ressort de mes recherches que l'apport de Goffman dans la compréhension des facteurs sociaux, politiques et médicaux qui ont contribué à la fermeture des asiles est indispensable pour juguler les crises à venir au sein du système de santé mentale et aider les ECTI à identifier les besoins immédiats pour leur bon fonctionnement en tant que nouvelle forme d'organisation psychiatrique communautaire.

\section{L'impact des guerres post-modernes sur les femmes et la construction identitaire de genre}

\section{Christiane Kheirallah \\ École de service social, Université d'Ottawa}

Les guerres post-modernes sont des guerres plus ambiguës que les deux premières guerres mondiales en ce qui a trait à la délimitation des participants, à la chronologie du conflit, à la détermination de la victoire et aux méthodes de combat. Dans ce genre de guerre, la plupart des victimes sont des civils et en particulier des femmes. Elles jouent des rôles divers dans les conflits et sont affectées par les événements selon le contexte dans lequel elles se trouvent et en relation avec leur identité de genre, d'ethnicité et de classe. Dans toutes les sociétés, il existe une 
dichotomie traditionnelle qui cause une inégalité de pouvoir entre les hommes et les femmes. Dans un contexte de guerre, ces différences sont accentuées car les hommes seront perçus comme guerriers et protecteurs et les femmes comme paisibles et vulnérables. Ces identités sont la cause de plusieurs sortes de violence envers les femmes, en particulier la violence sexuelle. Dans les guerres post-modernes, la violence sexuelle a été utilisée comme arme de guerre et ce n'est que récemment que la communauté internationale a pris des mesures pour contrer cette situation. De surcroît, les femmes sont les victimes de violence structurelle, institutionnelle, politique, domestique, psychologique et physique durant un conflit. Elles sont contraintes à se déplacer et à se réfugier, elles vivent des difficultés financières et connaissent des changements familiaux et personnels. Toutefois, certaines femmes arrivent à obtenir des gains durant la guerre. Elles transforment leurs rôles dans la sphère privée et publique. Elles s'impliquent dans des organisations féministes et humanitaires et participent à la vie politique. Cependant, plusieurs de ces transformations ne sont que temporaires. Une fois que la guerre est terminée, la société s'attend à ce que les femmes reprennent leurs anciens rôles. Ainsi, les actions des femmes sont analysées comme étant une extension de leurs rôles domestiques et nourriciers. Donc, les femmes arrivent à avoir des rôles divers et nouveaux durant un conflit armé, mais elles n'arrivent pas à transformer les identités que la société leur assigne.

\title{
Les parcours de vie de jeunes adultes ayant survécu au cancer. Étude exploratoire.
}

\author{
Janelle Lalonde \\ École de service social, Université d'Ottawa \\ Les progrès technologiques remarquables en matière de dépistage \\ et de traitement du cancer des dernières décennies ont favorisé la \\ survie des personnes atteintes de cette maladie. À ce jour, les
}


recherches menées pour comprendre les conséquences à long terme du cancer et de ses traitements sur les parcours de vie des individus sont peu nombreuses. Les jeunes présentent une catégorie particulière de ce point de vue puisqu'ils ont devant eux un avenir qui s'étend sur des plages de temps plus longues. De plus, la période de la vie qui suit l'adolescence est «sensible» à plusieurs égards: la poursuite des études, le choix d'une carrière, le départ du foyer parental et la mise en couple, sont autant de décisions et d'événements qui orientent les parcours de vie et qui ont habituellement lieu au début de la vingtaine. Ce mémoire vise donc à analyser les parcours et les difficultés rencontrées par des jeunes adultes survivantes et survivants du cancer. Notamment, nous cherchons à identifier les différences qui marquent les parcours de ces jeunes, les obstacles qui les ponctuent et les stratégies mises en œuvre pour y répondre. À cette fin, nous étudions tout particulièrement la formation scolaire, les relations amicales, l'identité, la santé physique et mentale, les relations familiales et de couple, la sexualité et l'emploi.

Pour répondre à ce questionnement, une enquête a été entreprise dans une perspective de recherche qualitative. La démarche visait à reconstituer les parcours depuis la fin des traitements et de saisir le sens que les individus y accordent. La recherche a été conduite auprès de six jeunes adultes ayant survécu à un cancer et ayant terminé leurs traitements depuis au moins un an. Les résultats montrent que les jeunes interviewés ne vivent pas les étapes transitoires vers la vie adulte d'une manière très différente de l'ensemble des jeunes, bien que certains admettent avoir eu à «se rattraper». Néanmoins, le cancer a eu des effets importants sur certaines dimensions de l'identité de ces jeunes. Malgré certaines difficultés, tous sont parvenus, à divers degrés, à s'ajuster positivement aux conséquences du cancer et de ses traitements grâce à leur sentiment de maîtrise de soi, leur optimisme et leurs relations avec des proches. Sur cette base, le mémoire soulève des pistes pour la recherche et l'intervention auprès des jeunes ayant survécu au cancer. 


\title{
Les soins à domicile en Ontario : les enjeux de la concurrence encadrée
}

\author{
Nadia Lauzon \\ École de service social, Université d'Ottawa
}

Après son élection au gouvernement ontarien en 1995, le Parti conservateur d'obédience néolibérale entreprend la restructuration du système de santé provincial, prônant la réduction du déficit budgétaire et la création d'un marché libre pour l'offre des services. Lors de cette restructuration, le gouvernement crée les Centres d'accès aux soins communautaires (C.A.S.C.) pour inclure les services à domicile comme service public. Ces C.A.S.C. sont des agences à guichet unique auquel les citoyennes et les citoyens peuvent s'adresser pour recevoir des soins à domicile. L'un des moyens choisis par le gouvernement pour diminuer les coûts entraînés par une demande de plus en plus forte pour les soins à domicile a consisté à mettre en place un système de concurrence encadrée. Les C.A.S.C. ne livrent pas eux-mêmes les soins, mais signent des contrats avec des compagnies privées ou des entreprises à but non lucratif, qui sont chargées de donner directement des soins.

Ce mémoire examine les répercussions d'un tel système de concurrence encadrée sur les usagères et les usagers et leurs familles, sur les compagnies privées, les entreprises à but non lucratif et sur la qualité des services, en se basant sur une étude des documents produits sur ce sujet. Ce mémoire distingue cinq grands enjeux. En premier lieu, la concurrence encadrée mène les gestionnaires à réduire la définition du terme «besoin» à un sens médical en vue de réduire l'accès aux soins à domicile. De plus, il transforme les usagères et les usagers ayant des droits en consommateurs de santé, sans pour autant leur donner un choix de services. Troisièmement, les familles sont perçues comme sources primaires de prise en charge d'une personne malade ou en perte d'autonomie avec tout ce que cela peut provoquer de difficultés. En quatrième lieu, la concurrence encadrée modifie profondément la façon dont les services de soins sont organisés et livrés, en 
créant une grande instabilité dans le personnel soignant et en réorganisant le travail de soin. Nous concluons que le système de gestion actuel des CASC met en danger la qualité des soins dispensés et, en les bureaucratisant, remet en question les valeurs propres au travail de soin (care) basé sur la qualité des rapports entre soignant et soigné.

\section{L'itinérance et les politiques sociales}

\section{Marie-Josée Legault}

École de service social, Université d'Ottawa

L'itinérance est un phénomène que l'on retrouve à travers le monde. Au Canada, elle prend de l'ampleur depuis les années quatre-vingt. Ce phénomène d'exclusion sociale affecte non seulement les adultes célibataires, mais de plus en plus, des familles avec de jeunes enfants, des adolescents, des autochtones, et des personnes immigrantes. Selon l'approche écologique, l'itinérance est un problème multidimensionnel qui se tisse entre les vulnérabilités personnelles d'un individu et les lacunes de la société. Ces lacunes peuvent être de plusieurs ordres et touchent notamment les structures du marché de l'emploi et du logement et les relations sociales. Cependant, il existe aussi des lacunes liées aux politiques sociales qui peuvent favoriser des situations d'exclusion.

En effet, selon des témoignages et des observations recueillis dans le cadre de ce mémoire, auprès de bénéficiaires de l'assistance sociale, d'intervenants communautaires et de gestionnaires locaux, la mise en oeuvre du programme d'assistance sociale Ontario au travail ainsi que l'orientation des politiques qui soutiennent ce programme, contribuent à créer un environnement d'exclusion où les bénéficiaires font face à des obstacles pour obtenir et maintenir un logement. Ces obstacles concernent l'insuffisance de l'allocation pour le logement, les documents exigés pour obtenir un logement, les erreurs administratives, le manque d'information, 
la méconnaissance du processus d'expulsion et l'insatisfaction liée au programme de démarrage communautaire. Les difficultés que posent ces obstacles associés au programme Ontario au travail peuvent même nuire à l'intégration des individus qui cherchent à mettre fin à leur situation d'itinérance. Des recommandations issues de la démarche de recherche sont également énumérées et commentées.

\section{La nouvelle économie sociale : une stratégie de survie pour la Péninsule acadienne?}

\section{Isabelle Légère}

École de service social, Université d'Ottawa

Dans la Péninsule acadienne, la pauvreté est une partie intégrante de la réalité de nombreuses familles et communautés puisque cette région connaît un sous-développement important. Étant donné l'intérêt de l'économie sociale comme stratégie possible de renouvellement économique au sein d'une communauté, nous nous sommes intéressés à sa pertinence pour une région telle que la Péninsule acadienne. Notre mémoire comporte donc un premier objectif qui consiste à explorer les éléments clés propres à la nouvelle économie sociale et un deuxième qui questionne la pertinence et l'utilité de celle-ci comme avenue de développement durable pour la Péninsule acadienne. La présente étude s'intéresse donc aux implications de la nouvelle économie sociale dans le contexte de la Péninsule acadienne.

Notre recherche s'appuie sur une analyse de contenu de vingtneuf articles scientifiques sur la nouvelle économie sociale, publiés depuis 1995. L'analyse des articles a permis de dégager certains des éléments clés de l'économie sociale, notamment ceux liés à l'empowerment individuel et collectif, au partenariat, à la communauté et à la création d'emplois et d'en approfondir le sens. 
Au terme de cette première analyse, en tenant compte des principes fondamentaux de l'économie sociale et de leur signification, nous avons formulé des questions qui ouvrent la porte à des pistes de réflexions sur les initiatives d'économie sociale entreprises dans la Péninsule acadienne. Nous avons finalement formulé quelques suggestions sur les plans de la pratique, des politiques sociales et de la recherche.

\title{
Les besoins en matière de santé des Franco- Ontariennes : analyse de dix documents portant sur la santé en Ontario français
}

\begin{abstract}
Renée C. Mallet
École de service social, Université d'Ottawa

L'objet de ce mémoire est d'analyser la façon dont certaines études sur la santé des francophones ont évalué les besoins des FrancoOntariennes. Pour ce faire, une analyse de contenu a été effectuée sur dix recherches faites en Ontario sur les besoins en matière de santé. Une lecture approfondie et répétée de ces documents a permis de reconnaitre les lacunes relatives à l'identification des besoins des Franco-Ontariennes dans les études et les recherches dirigées en Ontario. Le mémoire vise à appliquer les compétences analytiques spécifiques à la profession du service social afin de mieux comprendre comment sont définis les besoins des FrancoOntariennes en matière de santé. Une analyse des études de besoins permet aussi aux travailleuses et travailleurs sociaux de constater l'impact et les enjeux de telles recherches effectuées fréquemment au sein des organismes sociaux et communautaires.
\end{abstract}




\section{La contribution du service social dans le développement du Sénégal : regard sur les causes endogènes du sous-développement}

\section{Léna Diamé Ndiaye}

École de service social, Université Laurentienne

Notre étude porte sur le développement et la culture au Sénégal. Plus particulièrement, il s'agit de la contribution du service social dans le développement à travers un regard sur les causes endogènes du sous-développement. Elle tente de comprendre les mécanismes du sous-développement afin d'élaborer des stratégies de développement pour l'Afrique en général et le Sénégal en particulier. Ce développement aura en son centre l'émergence d'une culture moderne.

L'analyse des causes endogènes du sous-développement a été souvent négligée. Nous avons voulu, à travers cette étude, réhabiliter cette approche. Réfléchir sur les comportements et les attitudes des acteurs du système nous paraît essentiel pour comprendre le phénomène. Nous partageons avec la majorité des experts que les causes exogènes liés au sous-développement, tels que l'héritage du colonialisme, l'impérialisme et la globalisation des marchés, sont déterminantes dans la compréhension de la situation actuelle des pays comme le Sénégal. Mais nous considérons qu'après quarante années de recherche de solutions, d'autres dimensions du problème doivent être explorées, comme celles de l'analyse des causes endogènes.

Il s'agit pour nous de montrer l'existence de comportements plus positifs liés à une culture moderne, comme la méthode, l'organisation, la transparence dans la gestion des affaires, la discipline au travail, le «désenchantement du monde» et de comportements négatifs issus des dysfonctionnements de la société sénégalaise, pouvant pour les premiers impulser le développement et pour les seconds entraver son processus. Notre corpus théorique 
s'est construit, pour l'essentiel, à partir des travaux de Boudreau, Kabou, Hilan, Dumont, Weber, Muchembled, Traoré.

La contribution du service social va se greffer, à travers les solidarités, dans le processus de transformation sociale dont le moteur sera l'éducation. Une scolarisation pour tous sera au centre de la stratégie à initier par l'État pour faire émerger de nouveaux comportements. Les mutations sociales, économique et politiques sont tributaires de la transformation des valeurs et la formation d'une nouvelle conception du monde. Il s'agit pour le travailleur social, de jeter un regard sur les causes endogènes, et dans ce cadre, nous n'avons pas la prétention d'apporter des réponses complètes et encore moins définitives.

\section{Le processus d'agrément au Centre de santé communautaire d'Hamilton et du Niagara}

\section{Florence Ngenzebuhoro}

École de service social, Université Laurentienne

Les Centres de santé communautaire passent régulièrement par un processus d'agrément Ce dernier consiste en une révision complète des politiques et des pratiques d'un centre de santé au niveau de cinq composantes : la gouvernance, la gestion et les pratiques administratives, les programmes et les services et, la capacité communautaire et la recherche.

Le stage effectué portait sur le processus d'agrément du Centre de santé communautaire de Hamilton-Niagara $(\mathrm{CSCH} / \mathrm{N})$ avec une attention particulière au concept de renforcement des capacités communautaires. Les buts identifiés dans le projet de stage étaient les suivants : comprendre le fonctionnement du processus d'agrément, identifier les défis et les succès d'un tel processus, cerner le rôle de la coordinatrice des programmes dans ce processus et, enfin, déterminer si le $\mathrm{CSCH} / \mathrm{N}$ se qualifie au processus d'agrément, dans quel cas, il répondrait aux critères établis dans le Manuel pour «Bâtir des organismes plus sains» (BOS). 
J'ai d'abord fait une présentation générale des activités menées par le CSCH/N.J'ai ensuite effectué une recherche approfondie sur le concept de capacité communautaire, en établissant les liens entre la théorie et les principes du manuel BOS et la pratique de l'intervention communautaire du $\mathrm{CSCH} / \mathrm{N}$. J'ai aussi abordé la question des défis et des succès du renforcement des capacités communautaires du $\mathrm{CSCH} / \mathrm{N}$. J'ai soumis une réflexion sur les avantages que j'ai tirés de ce stage sur les plan professionnel et personnel et, finalement, j'ai présenté des recommandations qui pourront aider le $\mathrm{CSCH} / \mathrm{N}$ à consolider sa vision sur le renforcement des capacités communautaires.

\section{Le suicide chez les jeunes : analyse de la littérature et d'un programme québécois de prévention}

\section{Sylvie Paiement}

École de service social, Université d'Ottawa

Au cours des dernières décennies, le taux du suicide chez les jeunes a augmenté de façon dramatique au Canada et plus particulièrement au Québec.Vu cette augmentation, le gouvernement québécois a établi, en 1998, une politique d'action face au suicide ayant pour but de freiner de $15 \%$, au cours des cinq années subséquentes, l'incidence suicidaire au Québec. Où en sont, aujourd'hui, les connaissances sur cette problématique ? Le but de ce mémoire est d'explorer, dans les écrits, les connaissances et les enjeux liés à la question du suicide chez les jeunes. À partir de l'ensemble des thèmes se dégageant de la littérature, nous avons analysé et critiqué la pertinence, pour l'intervention auprès des jeunes, de la politique "Stratégie d'action face au suicide» du gouvernement québécois. Ce mémoire comporte donc deux volets: une présentation des résultats d'une analyse documentaire des écrits scientifiques sur le suicide chez les jeunes et l'analyse de la pertinence, en lien avec cette problématique, de la politique québécoise d'action face au suicide. 
Nous avons d'abord exposé et conceptualisé la problématique en tentant de situer qui sont les jeunes aujourd'hui et d'aborder certains des défis auxquels ils sont confrontés. Après avoir présenté un aperçu historique et épidémiologique du suicide au Canada et au Québec, nous avons ensuite cerné certaines perspectives d'analyse des facteurs du suicide. Dans un deuxième temps, une analyse documentaire des articles de recherche a permis l'identification d'éléments retenus dans la compréhension et l'explication du suicide chez les jeunes. Ainsi quatre thèmes semblent importants à considérer dans l'étude de ce problème, notamment le contexte social, familial et communautaire, les enjeux associés au genre et à l'identité sexuelle, les déterminants psychologiques individuels et finalement les enjeux liés à la violence et à la victimisation. En dernier lieu, en nous appuyant sur les connaissances se dégageant de la littérature, nous avons analysé la pertinence, pour les jeunes, de la politique québécoise d'action face au suicide. Cette analyse nous a permis d'élaborer des pistes de réflexion sur la pratique, sur la recherche et sur les politiques sociales en matière de prévention et d'intervention dans la problématique du suicide chez les jeunes.

\title{
Le communautaire et l'économie sociale peuvent-ils être une alternative utile pour les ex-psychiatrisés-es?
}

\begin{abstract}
Hélène Petit
École de service social, Université d'Ottawa

Ce mémoire comporte une recension des écrits sur le communautaire en santé mentale, son histoire et ses caractéristiques, afin de mieux cerner ce qui le définit et le caractérise. Pour ce faire nous utilisons le modèle de White et Mercier qui subdivise les ressources communautaires en santé mentale en deux grandes catégories : les ressources intermédiaires et les ressources alternatives. Cette démarche permet également de mettre en lumière l'importance que le communautaire occupe sur la scène politique au Québec.
\end{abstract}


Dans un deuxième temps nous retraçons également l'historique de l'économie sociale à travers la notion d'empowerment en évoluant vers la nouvelle économie sociale et ses caractéristiques contemporaines. Puis nous regardons comment se tissent les liens entre le communautaire et l'économie sociale, en en faisant valoir l'intérêt mais aussi les enjeux et les pièges.

Nous concluons par ce que nous y voyons comme implications autant pour la pratique, que pour la recherche-action.

\section{L'inceste maternel : fantasme ou réalité?}

\section{Myriam Rousseau}

École de service social, Université Laurentienne

Ce mémoire est une réflexion sur la question de l'inceste, plus spécifiquement de l'inceste maternel. L'inceste maternel est décrit comme étant un phénomène rare. Bien qu'on n'ait guère entendu parler des femmes abuseurs sexuels dans le domaine des sciences sociales, ce phénomène prend une ampleur grandissante, tant dans la recherche que dans la pratique du service social.Toutefois, malgré un regain d'intérêt de la part des chercheurs et des cliniciens, cette réalité que représente la femme abuseur sexuel a largement été négligée, tout particulièrement depuis que l'on s'occupe de la question de l'abus sexuel des enfants. Pourtant, cette question soulève aujourd'hui de nombreux débats et questionnements, en particulier lorsqu'il s'agit de ses implications théoriques et à propos de l'intervention appropriée à prévoir.

Nous en sommes venus à conclure que la reconnaissance de l'inceste maternel est difficile parce que sa reconnaissance sociale évoque l'impensable, l'inimaginable, voire la reconnaissance du pis social. En fait, cette reconnaissance porte un dur coup à l'idéologie et aux croyances propagées par le féminisme, depuis deux générations maintenant, selon lesquelles la femme n'est qu'un objet, une victime de l'agression masculine, jamais un sujet porteur de la violence envers l'autre. 
Nous sommes encore très loin de posséder une connaissance suffisante à propos des mères incestueuses. Il y a d'énormes lacunes à combler en matière d'information publique, de conscientisation, de sensibilisation, de recherches et de programmes de traitement, particulièrement en ce qui concerne la formulation d'un discours plus inclusif sur la violence perpétrée par les femmes.

C'est dans ce contexte que l'objectif de cette recherche a été et demeure de souligner, d'aider et de briser le silence qui entoure le tabou que représentent les mères incestueuses, et d'arriver à faire reconnaitre, sur ses bases véritables, l'existence de ce phénomène. 